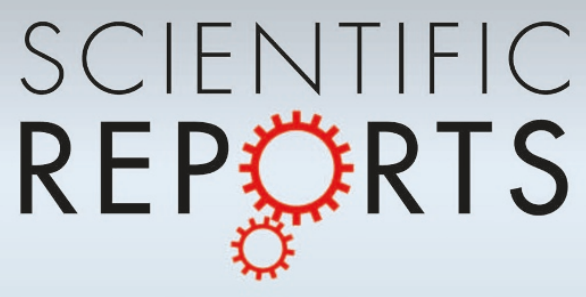

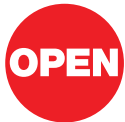

SUBJECT AREAS:

NANOCAVITIES

NANOPORES

MICRO-OPTICS

STRUCTURAL PROPERTIES

Received

13 November 2012

Accepted

25 February 2013

Published

19 March 2013

Correspondence and requests for materials should be addressed to S.W.D. (dusw@ust.hk) or Z.K.T. (phzktang@ ust.hk)

\section{Estimating Atomic Sizes with Raman Spectroscopy}

\author{
Dingdi Wang', Wenhao Guo', Juanmei Hu', Fang Liu'², Lisheng Chen², Shengwang Du' \& Zikang Tang'
}

'Department of Physics, The Hong Kong University of Science and Technology, Clear Water Bay, Kowloon, Hong Kong, China,
${ }^{2}$ Wuhan Institute of Physics and Mathematics, Chinese Academy of Sciences, Wuhan 430071, China.

We demonstrate a technique to determine the Van der Waals radius of iodine atoms using Raman spectroscopy. The iodine diatomic molecules are diffused into the nano-scale channels of a zeolite single crystal. We found their polarized Raman spectroscopy, which corresponds to iodine molecule's vibrational motion along the direction of molecular axis, is significantly modified by the interaction between the iodine molecules and the rigid frame of the crystal's nano-channels. From the number of excitable vibration quantum states of the confined iodine molecules determined from Raman spectra and the size of the nano-channels, we estimate the iodine atomic radius to be $2.10 \pm 0.05 \AA$. It is the first time that atomic sizes, which are far beyond the optical diffraction limit, have be resolved optically using Raman spectroscopy with the help of nano-scale structures.

toms, with radii of about angstrom $\left(\AA, 10^{-10} \mathrm{~m}\right)$, are the building blocks of our nature physical world. However, they are too small to be seen with visible light. A conventional optical microscope can only tell spots with a size larger than half of the optical wavelength, which is more than $2,000 \AA$, limited by the optical diffraction ${ }^{1}$. A scanning tunneling microscope (STM) is able to reach a subatomic resolution ${ }^{2}$, but it is expensive and also has some certain limitations. Here we show that atomic sizes can be resolved optically with Raman spectroscopy. We load iodine molecules into nano-channels of a zeolite single crystal, and find their Raman spectroscopy is significantly modified by the interaction between the iodine molecules and the rigid frame of the crystal's nano-channels. The number of excitable vibration quantum states of such a diatomic molecule is limited by the size of the nano-channel. We determine the radius of an iodine atom from the data of Raman spectroscopy. Our work not only demonstrates a spectroscopy probe for measuring the atomic sizes with nanochannel, but also may lead to practical applications in nano science and technology, such as designing, fabricating, and diagnosing nano materials and structures in the level of single atoms and molecules.

An atom consists of a central nucleus surrounded by a cloud of electrons, which determines the size or the boundary of the atom. Thus the atomic size plays an important role in the physical and chemical properties of the atom, as shown in the periodic table. It is well known that the atomic or molecular size affects the real gas state equation. A deviation from the ideal gas model was first pointed out by Van der Waals ${ }^{3}$. For this reason, we often call the radius of a spherical atom as the Van der Waals radius. While the electron distribution of the hydrogen atom can be precisely calculated following quantum mechanics, it is extremely difficult to do so for multi-electron atoms. Experimentally, besides the STM, there have been some indirect methods to estimate the scale of atomic sizes, such as X-ray diffraction and neutron diffraction ${ }^{4-9}$.

In this letter, we focus on determining the Van der Waals radius of the atoms in an iodine molecule. As the atoms are in the electronic ground state configuration, we can treat them as two hard spheres with a radius of $r_{a}$ and internuclear separation of $r$. Our modeled systems are schematically illustrated in Figure 1. In free space, the diatomic interaction can be described by the Morse potential ${ }^{10}$, which allows a large number of discrete vibration quantum states, as shown in the right panel of Figure 1a. However, the situation is different when the iodine molecule is loaded into a cylindrical cage with an internal diameter $2 R$ (here $R$ is the radius) slightly larger than the size of the ground state molecule. At higher vibration states, the vibration amplitude increases such that at a certain energy level the atoms start to collide with the cage wall (Figure $1 \mathrm{~b}$ ). The vibration states at higher energies are blurred due to the vibration energy dissipation from the molecule to the framework of the wall, as illustrated in the right panel of Figure 1b. As a result, Raman transitions to higher vibration states, which exist in the free space, disappear when the molecule is trapped inside the cage. Thus we may be able to determine the iodine atomic size from the data of Raman spectroscopy if the cage dimension is precisely known.

With a given diatomic interaction potential $U(r)$ inside the nano cage, we can determine the outer turning position at $r_{t}$ by $U\left(r_{t}\right)=E$ for a specific vibrational state with the energy eigenvalue $E$ which can be obtained from 
(a)

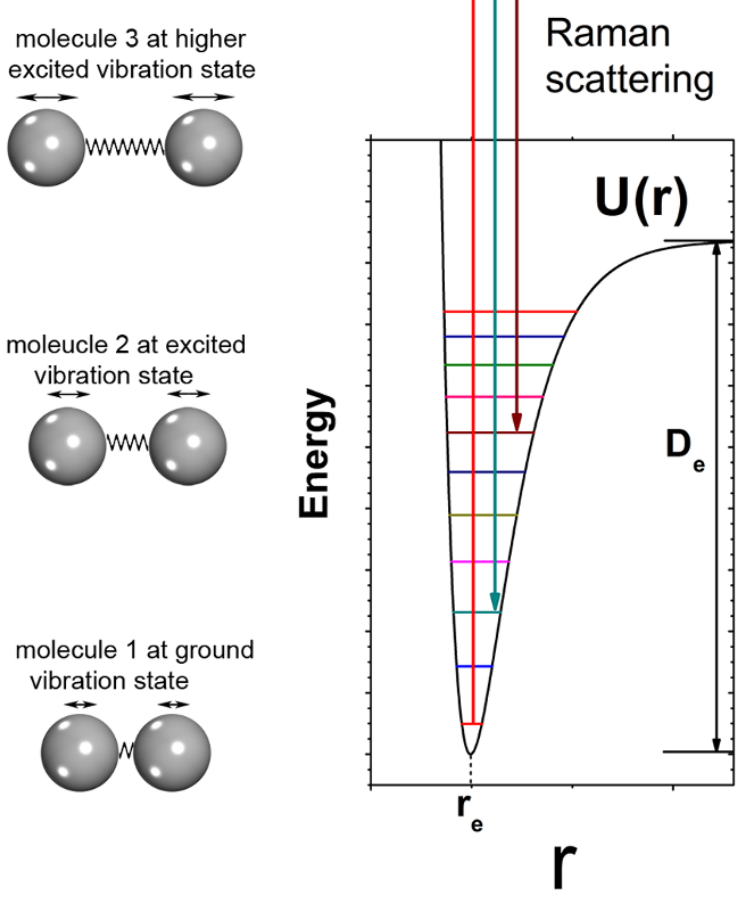

(b)

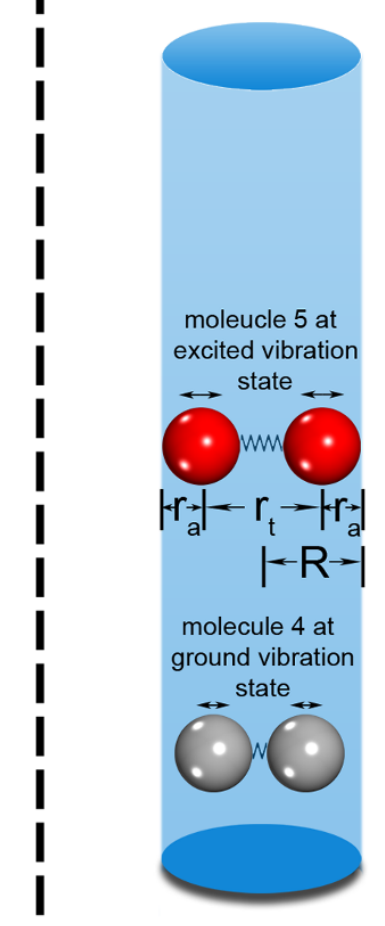

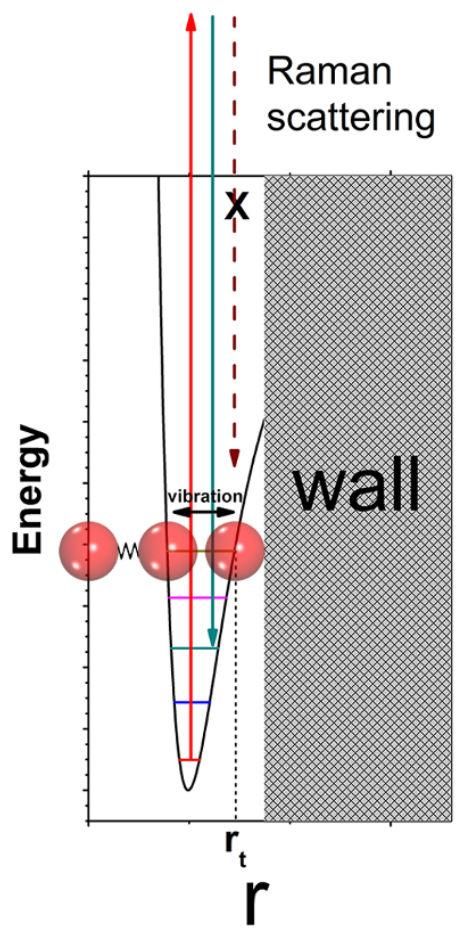

Figure 1 Schematics of diatomic molecules and two-photon Raman transitions between vibration levels at the electronic ground state.

(a) The left panel depicts in free space a molecule at ground vibration energy level and two molecules at higher vibration energy levels, and the right panel represents the molecular Raman transitions in the free space. (b) Two molecules confined in a rigid molecular nano channel are illustrated in left panel. The right panel describes molecular Raman transitions in the nano channel. The two atoms of the diatomic molecule at a specific vibrational state would vibrate between the inner turning position (smallest internuclear separation) and outer turning position (largest internuclear separation) of that state. When diatomic molecules are put into a rigid cylindrical cage, if the internuclear separation $r_{t}$ of the outer turning position plus the atomic diameter $2 r_{a}$ is larger than the size of the cage, in other words $r_{t}+2 r_{a} \geq 2 R$, these vibration states will be blurred due to the vibration energy dissipation from the molecule to the framework of the wall, which will be revealed by Raman spectroscopy. If the internal radius $R$ of the cylindrical cage is well known, the atomic radius $r_{a}$ of the confined molecule can be calculated through $r_{a}=R-r_{t c} / 2$.

the Raman shift. It is well known that, at a high vibrational energy state, the probability density is concentrated at these classical turning positions, where the atom spends most of its time with minimum speed $^{11}$. Meanwhile, the atoms at their outer turning positions are much closer to the wall of the nano cage and have higher probability in colliding incoherently with the wall. As the outer turning positions approach to the wall, the two-photon Raman transition probability reduces. Therefore, maintaining a free-state-like vibrational state requires $r_{t}+2 r_{a} \leq 2 R$. The molecule 5 in Figure $1 \mathrm{~b}$ represents such a critical case where the sphere of the atom coincides with the wall: $r_{t c}+2 r_{a}=2 R$, i.e., $r_{a}=R-r_{t c} / 2$.

\section{Results}

We use aluminophosphate $\left(\mathrm{AlPO}_{4}\right)$ porous single crystals as the "molecular ruler", which have been widely adopted as host template platforms since their ultra-small opening nano-channels are suitable to trap small guest molecules ${ }^{12-21}$. AlPO $\mathrm{PO}_{4}$ single crystals are transparent from ultraviolet to near-infrared, which are appropriate for the optical investigation of the trapped guest molecules ${ }^{18-23}$. We had introduced iodine molecules into the nano-channels of $\mathrm{AlPO}_{4}-5$ (AFI) and $\mathrm{AlPO}_{4}-11$ (AEL) single crystals and investigated the vibrational energy level separations of the confined iodine molecules in our previous work ${ }^{24}$.

The framework structures of AFI and AEL crystals have been well determined by X-ray diffraction and neutron diffraction experiments ${ }^{25-27}$. The main channel (cylindrical cage) of AFI crystal consists of twelve alternating $\mathrm{AlO}_{4}$ and $\mathrm{PO}_{4}$ tetrahedrons, leading to a circular cross section. The diameter of the space enclosed by channel framework is about 7.3 $\AA$. While the main channel of AEL crystal is composed of ten alternating $\mathrm{AlO}_{4}$ and $\mathrm{PO}_{4}$ tetrahedrons with $7.2 \AA$ major and $5.3 \AA$-minor elliptical enclosed space. The iodine molecules are oriented along the major axis not the minor axis in AEL channels. This configuration is confirmed by molecular dynamics simulation as well as polarized Raman spectroscopy ${ }^{20,21,28}$.

The polarized Raman spectra of iodine molecules in free space, accommodated in AFI ( $\left.\mathrm{I}_{2} @ A F I\right)$ and in AEL $\left(\mathrm{I}_{2} @ A E L\right)$ crystals are shown in Figure 2a, 2b and 2c, respectively. The Raman scattering is polarization independent for free-standing iodine molecules, reflecting their isotropic characteristics. In contrast, the Raman scattering of the $\mathrm{I}_{2} @ \mathrm{AEL}$ is strongly polarized along the major elliptical axis direction, while the Raman polarization property of the $\mathrm{I}_{2} @ A F I$ is in between vapor phase iodine and $\mathrm{I}_{2} @ A E L$. More than fifty Raman peaks are observable for the free-standing iodine molecules. The intensity of the overtones decreases slowly. In comparison, the intensities of the overtones of the confined iodine molecules decrease rapidly. In the Raman spectra (Figure 2), only fourteen and ten overtones are observable in $\mathrm{I}_{2} @ A F I$ and $\mathrm{I}_{2} @ A E L$ crystals. We attribute these phenomena to the confinement effects on the iodine molecules. The vibration movements of the confined molecules are gradually influenced by the framework atoms of the wall. At higher vibration states, the magnitude of vibration decreases dramatically due to the vibration energy dissipation to the framework atoms, and the vibration is gradually undetectable in the Raman spectrum. 


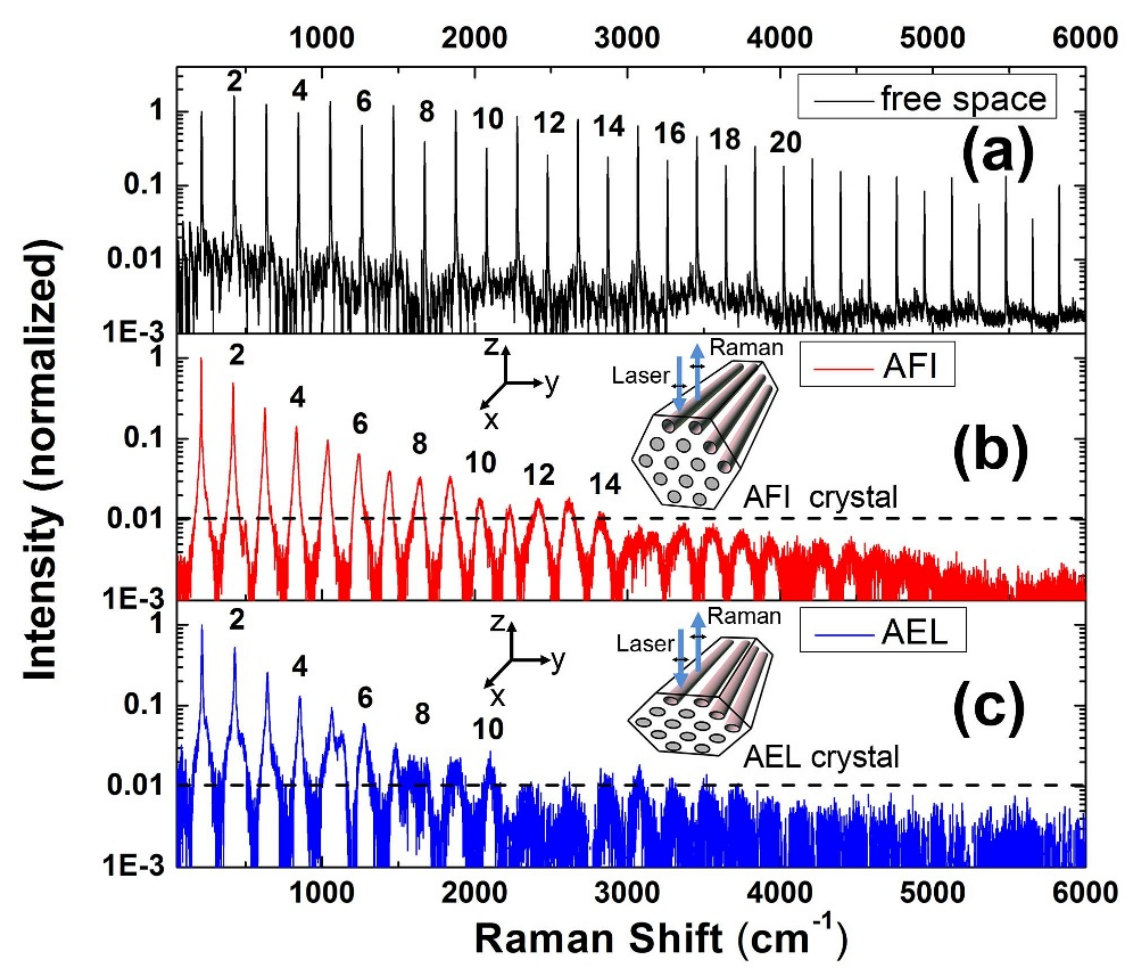

Figure $2 \mid$ Polarized Raman spectra of iodine molecules in free space, inside an AFI crystal and an AEL crystal for excitation with a $514.5 \mathrm{~nm}$ laser line. (a) In free space, more than 30 peaks are clearly observed with iodine molecules. (b) For iodine molecules trapped inside the AFI crystal, there are about 14 monotonously decreasing peaks. (c) With enclosed molecules in the AEL crystal, only about 10 peaks are identified. It is clearly seen that, in b and c, the intensity of the peaks of confined iodine molecules presented an exponential decay nature. These phenomena are attributed to the different degree of channel framework's confinement on the vibrational motion of the embedded iodine molecules. The configurations of Raman scattering experiment are illustrated in the insets of (b) and (c). The incident excitation laser line is polarized along the $y$-axis and propagates along the negative $\mathrm{z}$-axis, while the back-scattering Raman signals propagates along the positive z-axis and are polarized along the $y$-axis. The AFI and AEL crystals are oriented such that their channels' direction parallels the $\mathrm{x}$-axis.

We define the point of the vibration magnitude down to $-20 \mathrm{~dB}$ or 0.01 as the maximum reachable boundary of the diatomic vibration. From the Raman spectra shown in Figure 2, this boundary is determined at the vibration state of $v=10 \pm 1$ for $\mathrm{I}_{2} @$ AEL crystals, and $v=14 \pm 1$ for $\mathrm{I}_{2} @$ AFI crystals, respectively. The magnitude of the molecular vibration would eventually be damped to zero if the vibration is reaching or overcrossing the vibration boundary.

The vibration potential at electronic ground state can be drawn from the overtones frequencies showed in the Raman spectra (Figure 2). We represent the internuclear potential with a Morse potential

$$
V(r)=D_{e} e^{-2 a\left(r-r_{e}\right)}-2 D_{e} e^{-a\left(r-r_{e}\right)}+D_{e}
$$

which is determined by three parameters $D_{e}, a$, and $r_{e}$, where $r_{e}$ stands for the equilibrium separation, $D_{e}$ denotes the dissociation energy, and $a$ determines the "width" of the potential ${ }^{10}$, as shown in the right panel of Figure 1a. The vibrational energy eigenvalues can be expressed as $E(v)=\omega_{e}\left(v+\frac{1}{2}\right)-\frac{\omega_{e}^{2}}{4 D_{e}}\left(v+\frac{1}{2}\right)^{2}, \quad$ where $\omega_{e}=\frac{a}{2 \pi}\left(\frac{2 D_{e}}{\mu}\right)^{\frac{1}{2}}$ denotes the classical vibrational frequency $(\mu$ stands for the reduced mass of the molecule). We determine these parameters by best fitting the numerically calculated vibrational energy spectrum to the Raman shifts shown in Figure 2. For freestanding iodine molecule, it is well determined that $\omega_{e}=$ $214.548 \mathrm{~cm}^{-1}, a=1.52270 \AA^{-1}$, and $D_{e}=18681.4 \mathrm{~cm}^{-129}$. The value $r_{e}=2.665 \AA$ can be get from rotational spectrum ${ }^{29}$. Because the rotational spectrum was not resolved in the confined state, we set $r_{e}=2.665 \AA$ in three cases. For $\mathrm{I}_{2} @ \mathrm{AFI}$, we get $\omega_{e}=212.5 \mathrm{~cm}^{-1}, a=$
$1.759 \AA^{-1}$ and $D_{e}=1.373^{*} 10^{4} \mathrm{~cm}^{-1}$. The vibration boundary at $v=$ $14 \pm 1$ corresponds to the critical turning position $r_{t c}=3.02 \pm 0.01$ $\AA$, as illustrated in Figure $3 \mathrm{a}$. In addition, the data of the lattice parameters of the AFI and AEL single crystals were well established by $\mathrm{X}$-ray and neutron diffraction ${ }^{25-27}$. Here we take the radii of framework atoms according to Batsanov's values': $2.10 \AA$, $1.95 \AA$, and $1.55 \AA$ for aluminum, phosphorus and oxygen, respectively. From these data, we obtain the effective inner diameter of the enclosed nano channel to be $7.3 \AA \pm 0.1 \AA$, as shown in Figure $3 \mathrm{~b}$. Then we estimate the radius of the iodine atom to be $r_{a}=\left(2 R-r_{t c}\right) / 2=2.10 \AA \pm 0.05 \AA$.

The nano-channels in the AEL crystal are elliptical. The polarization Raman spectrum shows all the iodine molecules in its nano channels are aligned along the major axis not the minor axis. From the Raman shift of the overtones, we determined the parameters of the interatomic potential as: $\omega_{e}=217.6 \mathrm{~cm}^{-1}, a=1.678 \AA^{-1}$ and $D_{e}$ $=1.582 * 10^{4} \mathrm{~cm}^{-1}$. The vibration boundary is at $v=10 \pm 1$, thus we obtain its outer turning position $r_{t c}=2.94 \pm 0.01 \AA$ (Figure 4a). The inner major axis of the enclosed nano channel to is $7.2 \AA \pm 0.1 \AA$, as shown in Figure $4 \mathrm{~b}$. Using the major axis radius $R_{j}=3.6 \AA$ of the nano channel, we determine the atomic radius $r_{a}=R_{j}-r_{t c} / 2=2.10$ $\AA \pm 0.05 \AA$. This value is consistent with the one obtained from AFI crystals.

\section{Discussion}

Table 1 shows a comparison of the iodine atomic radius determined in this work with those reported in the literature. The radii reported in the literature are mainly determined from X-ray diffraction and neutron diffraction technologies. It can be seen that there is an approximate $10 \%$ uncertainty of iodine's radius. This is because 


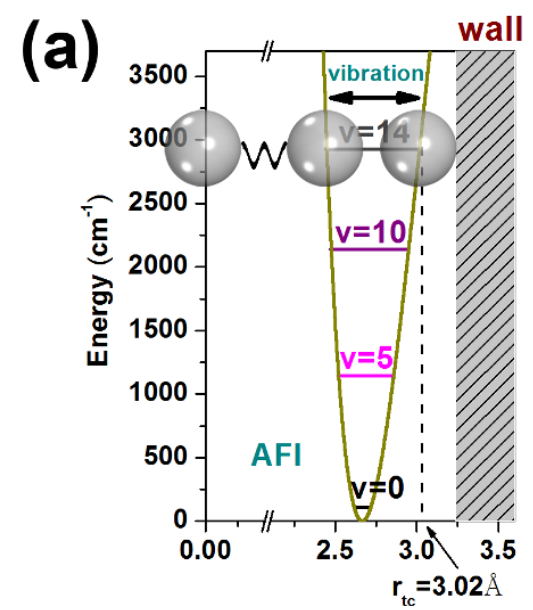

Internuclear Seperation $(\AA)$ (b)

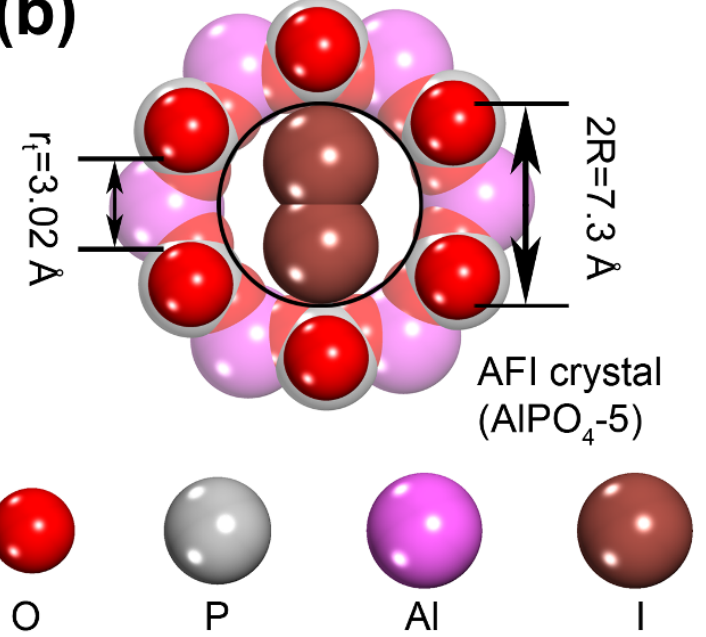

Figure $3 \mid$ The determination of atomic radius of iodine atoms confined in the AFI crystal. (a) Vibrational Morse potential curve of iodine molecule in AFI crystal. The energy levels of $v=0, v=5, v=10, v=14$ are plotted. The iodine molecule is plotted such that one atom is fixed at zero point, the other one vibrates between the two turning points of that level (where the state's energy coincides with the potential energy). The outer turning point of the last observable vibrational state $v=14$ can be identified from the potential curve, which is $r_{t c}=3.02 \AA$. We model that the iodine atoms would collide with walls at this position. (b) The instant moment of iodine atoms strike the framework of main channel atoms viewed from [001] direction. The internuclear distance of two iodine nuclei is set to $3.02 \AA$. The diameter of the space enclosed by channel framework is $7.3 \AA \pm 0.1 \AA$. Then we estimate the radius of the iodine atom to be $r_{a}=\left(2 R-r_{t c}\right) / 2=2.10 \AA \pm 0.05 \AA$.

the same atom in different molecular crystals would manifest slightly different radii. In fact, the atomic radius just depicts an imaginary sphere to represent the electron density distribution for each atom, the actual distribution may have a rather complicated shape rather than simple sphere. From Table 1 we can see that, generally speaking, the atomic radius of confined iodine atom determined from the Raman spectra are in good agreement with the values got from other methods.

In summary, we proposed and demonstrated an optical spectroscopy approach to estimate the atomic radius of iodine atom through a "molecular vibrational confiner". The confined iodine molecule at a vibrational state higher than a critical value will collide with the channel wall by dissipating vibration energy to the host framework atoms. As a result, the overtones to the higher vibration states disappear in the Raman spectra. With the known size of the nano channels, the Van der Waals radius of the iodine atom can then be determined from the number of excitable vibrational levels. This technique provides a novel method to "see" the size of atoms with a high precision. Our result may lead to practical applications in nano science and technology, as well as fundamental research in atomic, molecular, and optical physics.

\section{Methods}

The AFI and AEL single crystals were synthesized by hydrothermal method ${ }^{30,31}$. The as-synthesized AFI and AEL single crystals have typical dimensions of $120 \times 120 \times$

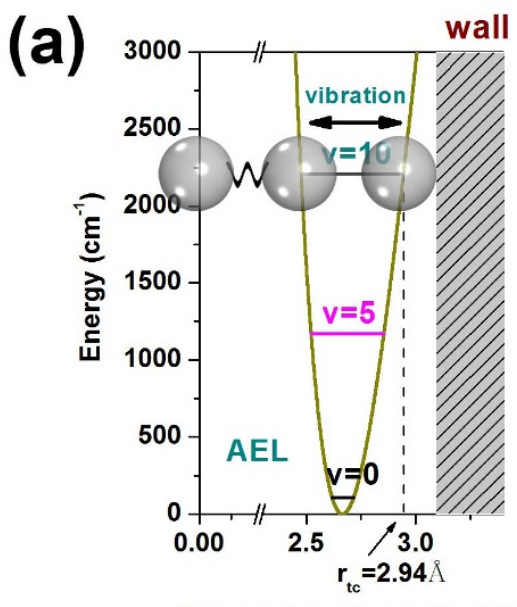

Internuclear Seperation $(\AA)$ (b)

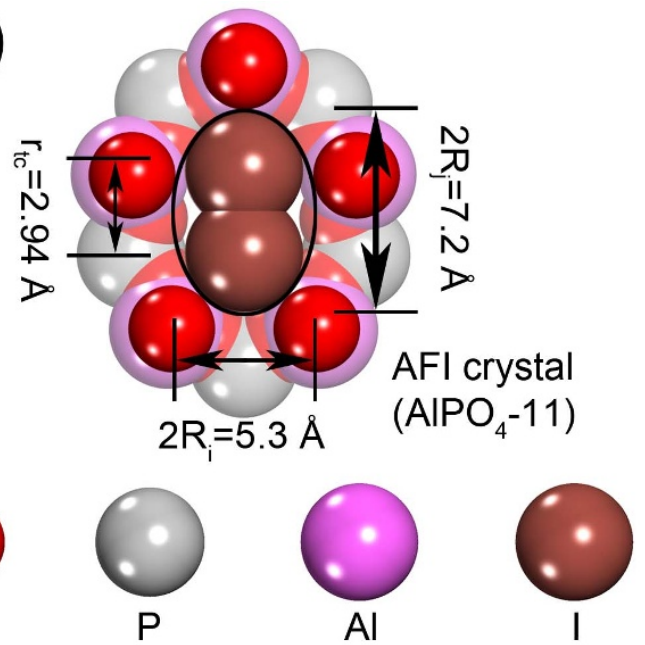

Figure $4 \mid$ The determination of atomic radius of iodine atoms confined in the AEL crystal. (a) Vibrational Morse potential curve of iodine molecule trapped inside the AEL crystal. The energy levels of $v=0, v=5, v=10$ are plotted. The iodine molecule is plotted such that one atom is fixed at zero point, the other one vibrates between the two turning points of that level (where the state's energy coincides with the potential energy). The outer turning point of the last observable vibrational state $v=10$ can be identified from the potential curve, which is $r_{t c}=2.94 \AA$. We model that the iodine atoms would collide with walls at this position. (b) The frame of iodine atoms hit the framework of main channel atoms view from [001] direction. The internuclear distance of two iodine nuclei is set to $2.94 \AA$. The major axis of the elliptical cross section is $7.2 \pm 0.1 \AA$. Using the major axis radius $R=3.6 \AA \pm 0.05 \AA$ of the nano channel, we determine the atomic radius $r_{a}=R-r_{t c} / 2=2.10 \pm 0.05 \AA$. 


\begin{tabular}{|lcc|}
\hline \multicolumn{3}{|l}{ Table 1 | Atomic radius of iodine determined by different methods } \\
author & radius (Å) & reference \\
\hline Bondi & 1.98 & 4 \\
Rowland & 2.03 & 5 \\
Gavezzotti & 2.10 & 6 \\
Zefirov & 2.14 & 7 \\
Pauling & 2.15 & 8 \\
Batsanov's crystallographic & 2.22 & 9 \\
Batsanov's equilibrium & 2.10 & 9 \\
inside AFI (this paper) & $2.10 \pm 0.05$ & \\
inside AEL (this paper) & $2.10 \pm 0.05$ & \\
\hline
\end{tabular}

$350 \mu \mathrm{m}$ and $10 \times 30 \times 70 \mu \mathrm{m}$, respectively. Iodine molecules were loaded into the nano-channels of AFI and AEL single crystals by a physical diffusion process. The AFI and AEL crystals were calcined at $580^{\circ} \mathrm{C}$ and $680^{\circ} \mathrm{C}$ respectively in $\mathrm{O}_{2}$ atmosphere for about 40 hours to remove the organic template molecules in the channels. Then the calcined crystals were sealed together with solid iodine source (BDH laboratory supplies, $>99 \%$ ) in a Pyrex tube under a vacuum of $10^{-2}$ mbar. Iodine molecules were physically diffused into the nano channels of the AFI and AEL single crystals at temperature $150^{\circ} \mathrm{C}$. The loading density of the iodine in the host crystals can be controlled by the weight ratio of the loaded solid iodine to the AFI (AEL) single crystals. We only focus on the low loading density samples in this experiment so as to ignore the intermolecular interaction.

The data were taken at room temperature using Jobin-Yvon T64000 micro-Raman spectrometer equipped with a liquid nitrogen cooled CCD detector in a backscattering configuration. A linearly polarized $514.5 \mathrm{~nm}$ beam output from an Innova $70 \mathrm{C}$ $\mathrm{Ar} / \mathrm{Kr}$ ion laser with a line width of $6 \mathrm{GHz}$ and a power of $30 \mathrm{~mW}$ was used as the excitation laser line. As shown in the insets of Figure $2 b$ and $2 c$, the incident excitation laser line is polarized along the $\mathrm{y}$-axis and propagates along the negative $\mathrm{z}$-axis, while the back-scattering Raman signals propagates along the positive $z$-axis and are polarized along the y-axis.

We use the vibration frequencies got from the first 12 peaks of the Raman spectrum of the iodine molecule confined in AFI crystal for plotting a Morse potential to represent the vibrational potential curve, and show it in Figure 3a. And we use the vibration frequencies got from the first 8 Raman peaks for extrapolating a Morse potential to represent the vibrational potential curve for the iodine molecule confined in AEL crystal, and show it in Figure 4a. The equilibrium interatomic distance is set to $2.665 \AA$, which is the same as the one of free iodine molecule determined from rotational spectrum ${ }^{29}$. The energies of the vibrational states are determined from Raman spectra, and then these turning points in potential curve are identified correspondingly.

1. Born, M. \& Wolf, E. Principles of Optics: Electromagnetic Theory of Propagation, Interference and Diffraction of Light Edn. 7. (Cambridge University Press, Cambridge; 1999).

2. Binnig, G. \& Rohrer, H. Scanning tunneling microscopy. IBM Journal of Research and Development 30, 355-369 (1986)

3. Poling, B. E., Prausnitz, J. M. \& O'Connell, J. The Properties of Gases and Liquids Edn. 5. (McGraw-Hill Professional, 2000).

4. Bondi, A. van der Waals Volumes and Radii. The Journal of Physical Chemistry 68, 441-451 (1964).

5. Rowland, R. S. \& Taylor, R. Intermolecular Nonbonded Contact Distances in Organic Crystal Structures: Comparison with Distances Expected from van der Waals Radii. The Journal of Physical Chemistry 100, 7384-7391 (1996).

6. Gavezzotti, A. The calculation of molecular volumes and the use of volume analysis in the investigation of structured media and of solid-state organic reactivity. Journal of the American Chemical Society 105, 5220-5225 (1983).

7. Zefirov, Y. V. Van der Waals atomic radii of group I-III metals. Russian Journal of Inorganic Chemistry 45, 1552-1554 (2000).

8. Pauling, L. The Nature of the Chemical Bond and the Structure of Molecules and Crystals: An Introduction to Modern Structural Chemistry, Edn. 3. (Cornell University Press, New York; 1960).

9. Batsanov, S. S. Van der Waals Radii of Elements. Inorganic Materials 37, 871-885 (2001).

10. Morse, P. M. Diatomic Molecules According to the Wave Mechanics. II. Vibrational Levels. Physical Review 34, 57-64 (1929).

11. Zare, R. N. Calculation of Intensity Distribution in the Vibrational Structure of Electronic Transitions: The $\mathrm{B}^{3} \Pi_{0+\mathrm{u}}-\mathrm{X}^{1} \Sigma_{0+\mathrm{g}}$ Resonance Series of Molecular Iodine. The Journal of Chemical Physics 40, 1934-1944 (1964).
12. Wang, N., Tang, Z. K., Li, G. D. \& Chen, J. S. Materials science: Single-walled $4 \AA$ carbon nanotube arrays. Nature 408, 50-51 (2000).

13. Demontis, P. et al. Dynamical Properties of Confined Water Nanoclusters: Simulation Study of Hydrated Zeolite NaA: Structural and Vibrational Properties. ACS Nano 2, 1603-1614 (2008).

14. Vohra, V.et al. Toward White Light Emission through Efficient Two-Step Energy Transfer in Hybrid Nanofibers. ACS Nano 4, 1409-1416 (2010).

15. Tsotsalas, M. M. et al. Encapsulating 111In in Nanocontainers for Scintigraphic Imaging: Synthesis, Characterization, and In Vivo Biodistribution. ACS Nano 4, 342-348 (2009).

16. Yang, S. Y. et al. Single-File Diffusion of Protein Drugs through Cylindrical Nanochannels. ACS Nano 4, 3817-3822 (2010).

17. Aydin, C. et al. Tracking Iridium Atoms with Electron Microscopy: First Steps of Metal Nanocluster Formation in One-Dimensional Zeolite Channels. Nano Letters 11, 5537-5541 (2011).

18. Tang, Z. K. et al. Resonant Raman Scattering of the Smallest Single-Walled Carbon Nanotubes. Physical Review Letters 101, 047402 (2008).

19. Jiang, F. Y. \& Liu, R. C. Incorporation of iodine into the channels of $\mathrm{AlPO}_{4}-5$ crystals. Journal of Physics and Chemistry of Solids 68, 1552-1555 (2007).

20. Zhai, J. P., Lee, H. F., Li, I. L., Ruan, S. C. \& Tang, Z. K. Synthesis and characterization of iodine molecular wires in channels of zeolite AEL single crystals. Nanotechnology 19, 175604 (2008).

21. Zhai, J. P., Li, I. L., Ruan, S. C., Lee, H. F. \& Tang, Z. K. Controlling the alignment of neutral iodine molecules in the elliptical channels of $\mathrm{AlPO}_{4}-11$ crystals. Applied Physics Letters 92, 043117 (2008).

22. Li, Z. M. et al. Polarized Absorption Spectra of Single-Walled 4 A Carbon Nanotubes Aligned in Channels of an $\mathrm{AlPO}_{4}-5$ Single Crystal. Physical Review Letters 87, 127401 (2001).

23. Guo, J. et al. Efficient Visible Photoluminescence from Carbon Nanotubes in Zeolite Templates. Physical Review Letters 93, 017402 (2004).

24. Guo, W., Wang, D., Hu, J., Tang, Z. K. \& Du, S. Raman spectroscopy of iodine molecules trapped in zeolite crystals. Applied Physics Letters 98, 043105 (2011).

25. Richardson, J. W., Pluth, J. J. \& Smith, J. V. Rietveld profile analysis of calcined $\mathrm{AlPO}_{4}-11$ using pulsed neutron powder diffraction. Acta Crystallographica Section B 44, 367-373 (1988).

26. Bennett, J. M., Cohen, J. P., Flanigen Edith, M., Pluth, J. J. \& Smith, J. V. in Intrazeolite Chemistry. Vol. 218 109-118 (AMERICAN CHEMICAL SOCIETY, 1983).

27. Pluth, J. J., Smith, J. V. \& Richardson, J. W. Crystal structure of the as-synthesized precursor (MnAl9)P10O40.cntdot. C6H16N to molecular sieve MnAPO-11. The Journal of Physical Chemistry 92, 2734-2738 (1988).

28. Hu, J. M., Zhai, J. P., Wu, F. M. \& Tang, Z. K. Molecular Dynamics Study of the Structures and Dynamics of the Iodine Molecules Confined in $\mathrm{AlPO}_{4}-11$ Crystals. The Journal of Physical Chemistry B 114, 16481-16486 (2010).

29. LeRoy, R. J. Molecular Constants and Internuclear Potential of Ground-State Molecular Iodine. The Journal of Chemical Physics 52, 2683-2689 (1970).

30. Jiang, F. Y., Zhai, J. P., Ye, J. T., Han, J. R. \& Tang, Z. K. Synthesis of large optically clear $\mathrm{AlPO}_{4}-5$ single crystals. Journal of Crystal Growth 283, 108-114 (2005).

31. Fu, L. et al. Synthesis of large silicon substituted $\mathrm{AlPO}_{4}-11$ single crystals. Microporous and Mesoporous Materials 137, 1-7 (2011).

\section{Acknowledgements}

D.W. wants to thank Lingfei Wang of Nanjing University and Haijing Zhang of HKUST for many meaningful discussions. This work was supported by Hong Kong CERGC Grants 603108 and 604210

\section{Author contributions}

S.D., Z.K.T. and L.C. proposed, designed, and directed the project. D.W., W.G., J.H. and F.L. carried out the experiment. All authors contributed to the final version of the manuscript.

\section{Additional information}

Competing financial interests: The authors declare no competing financial interests.

License: This work is licensed under a Creative Commons

Attribution-NonCommercial-NoDerivs 3.0 Unported License. To view a copy of this license, visit http://creativecommons.org/licenses/by-nc-nd/3.0/

How to cite this article: Wang, D.D. et al. Estimating Atomic Sizes with Raman Spectroscopy. Sci. Rep. 3, 1486; DOI:10.1038/srep01486 (2013). 